\title{
Efficient GRASP+VND and GRASP+VNS metaheuristics for the traveling repairman problem
}

\author{
Amir Salehipour • Kenneth Sörensen • \\ Peter Goos • Olli Bräysy
}

Received: 26 April 2010 / Revised: 29 December 2010 / Published online: 29 January 2011

(C) The Author(s) 2011. This article is published with open access at Springerlink.com

\begin{abstract}
The traveling repairman problem is a customer-centric routing problem, in which the total waiting time of the customers is minimized, rather than the total travel time of a vehicle. To date, research on this problem has focused on exact algorithms and approximation methods. This paper presents the first metaheuristic approach for the traveling repairman problem.
\end{abstract}

Keywords Traveling repairman problem · Minimum latency problem · Variable neighborhood descent · Variable neighborhood search · GRASP

MSC classification (2000) 90C27 · 90C59

\section{Introduction}

The traveling repairman problem (TRP), also called the minimum latency problem or the traveling deliveryman problem, is defined on a weighted graph $G=(V, E, w)$.

\footnotetext{
A. Salehipour · K. Sörensen $(\varangle) \cdot$ P. Goos

Operations Research Group ANT/OR, Faculty of Applied Economics,

University of Antwerp, Prinsstraat 13, 2000 Antwerp, Belgium

e-mail: kenneth.sorensen@ua.ac.be
}

\author{
A. Salehipour \\ e-mail: amir.salehipour@ua.ac.be \\ P. Goos \\ Erasmus School of Economics, Erasmus Universiteit Rotterdam, \\ Postbus 1738, 3000 DR Rotterdam, The Netherlands \\ e-mail: peter.goos@ua.ac.be \\ O. Bräysy \\ Agora Innoroad Laboratory, Agora Center, University of Jyväskylä, \\ P.O. Box 35, 40014 Jyväskylä, Finland \\ e-mail: olli.braysy@jyu.fi
}


The vertex set $V=\left\{v_{0}, \ldots, v_{n}\right\}$ represents a set of locations, that have to be visited, starting from $v_{0}$. With each edge $\left(v_{i}, v_{j}\right) \in E$, a weight $w\left(v_{i}, v_{j}\right)$ is associated, that represents the travel time between $v_{i}$ and $v_{j}$. Sometimes, an additional time $t_{k}$ is associated with each vertex $v_{k}$, to represent the time spent at this vertex. In the symmetric TRP, travel times between vertices are the same in both directions, i.e., $w\left(v_{i}, v_{j}\right)=w\left(v_{j}, v_{i}\right)$.

The objective of the TRP is to find an open Hamiltonian circuit, starting from $v_{0}$, that minimizes the total waiting time. The waiting time of a vertex, also called its latency, is defined as the time it takes to reach it. The TRP can be seen as a "customercentric" routing problem, because the objective function minimizes the total waiting time of all customers (vertices), rather than the travel time of the vehicle the customers is visited with. Besides the obvious applications in customer-centric routing (e.g., a doctor visiting patients, or a repairman visiting machines that require servicing), the TRP has applications in e.g., disk head scheduling. An example traveling repairman problem, including a (symmetric) distance matrix, can be found in Fig. 1.

The TRP is very similar to the traveling salesman problem (TSP) and several different mathematical programming formulations have been proposed [see, e.g., Fischetti et al. 1993; Sarubbi and Luna 2007; Méndez-Díaz et al. 2008]. On a metric space, the TRP is NP-hard (Blum et al. 1994) like the TSP but surprisingly, the TRP is much harder to solve or approximate. Polynomial time algorithms exist in a number of special cases, such as paths (Afrati et al. 1986; García et al. 2002) or trees of diameter 3 (Blum et al. 1994). A lot of research has gone into developing approximations for the TRP, and the current best approximation ratio is 3.59 (Chaudhuri et al. 2003). In contrast, the best-known approximation ratio for the metric TSP is 1.5, due to Christofides (1976). As stated by Méndez-Díaz et al. (2008), the TRP is a very hard problem to solve to proven optimality.

Several exact algorithms for the TRP exist, such as the dynamic programming algorithm of $\mathrm{Wu}$ (2000) or the much more efficient branch and bound algorithm of Wu et al. (2004). Due to the NP-hardness of the TRP, however, these approaches are unable to solve medium- and large-scale instances. The fastest method of Wu et al. (2004) requires $100 \mathrm{~s}$ to solve a problem with approximately 25 vertices, which makes this method faster and more powerful than an implementation of CPLEX on a standard formulation.

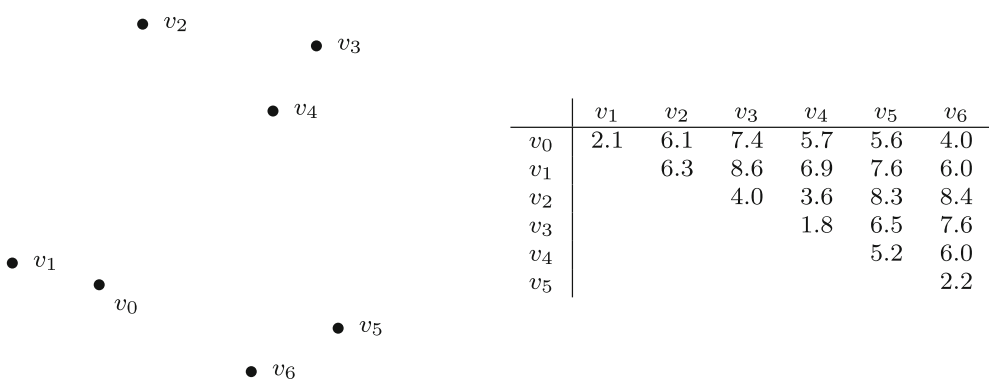

Fig. 1 Example traveling repairman problem and (symmetric) distance matrix 
Research on the TRP has focused almost exclusively on either approximation algorithms or exact methods and this paper presents the first metaheuristic approach for this problem. However, a limited number of metaheuristics have appeared since the development of our method. Most notably, the memetic algorithm of Ngeuveu et al. (2010) focuses on a capacitated version of the TRP, called the vehicle routing problem with cumulative demand.

Our GRASP+VND/VNS approach is a multi-start method in which each iteration consists of two phases: a greedy randomized construction phase and a variable neighborhood descent or variable neighborhood search improvement phase. The approach is tested on a number of instances, both randomly generated ones and instances from the TSP Library. ${ }^{1}$

GRASP (Greedy Randomized Adaptive Search Procedure) was introduced by Feo and Resende $(1989,1995)$. The basic idea of GRASP is to allow a controlled amount of randomness to overcome the myopic behaviour of a purely greedy heuristic. This method has been applied to a large number of combinatorial optimization problems, including routing problems such as the vehicle routing problem with time windows (Kontoravdis and Bard 1995). Although initial implementations of GRASP combined it with simple local search strategies, more recent work combines it with more advanced local search approaches.

Among those more advanced local search schemes is variable neighborhood search (VNS) (Mladenović and Hansen 1997; Hansen and Mladenović 1999, 2001b), a recent metaheuristic for combinatorial optimization. VNS is based on the principle of systematically exploring several different neighborhoods, combined with a perturbation move (called shaking in the VNS literature) to escape from local optima. Despite the fact that it has been developed only recently, variable-neighborhood search has been successfully applied to a wide variety of well-known and lesser-known optimization problems (Hansen and Mladenović 2001a,b). Several researchers have applied some form of VNS to (more or less academic) vehicle routing problems. Two examples are Cordone and Wolfler Calvo (2001), in which a VNS algorithm for the vehicle routing problem with time windows is developed, and Crispim and Brandao (2001), who present a VNS approach to the vehicle routing problem with backhauls.

Variable neighborhood descent (VND) is essentially a simple variant of VNS, in which the shaking phase is omitted. Therefore, contrary to VNS, VND is usually completely deterministic. The combination of GRASP and VND is not new: Ribeiro and Vianna (2003), for example, applied it to the phylogeny problem.

It is important however to note that the concept of multiple neighborhood search (i.e., using more than one neighborhood type) is broader than the one proposed in variable neighborhood search and that a large number of non-VNS approaches use different neighborhood types. In the list of best-performing approaches for most vehicle routing problems, the idea of multiple neighborhood search is overwhelmingly present. In their survey article on metaheuristics for the vehicle routing with time windows, Bräysy and Gendreau (2005) find that a large majority of tabu search approaches and genetic algorithms use more than one type of neighborhood.

\footnotetext{
1 http://comopt.ifi.uni-heidelberg.de/software/TSPLIB95/.
} 
The rest of this paper is organized as follows. In Sect. 2, we discuss our GRASP+VND as well as our GRASP+VNS algorithms for the TRP. To evaluate the performance of these approaches, we discuss an upper bound and a lower bound in Sect. 3. A set of benchmark instances is generated in Sect. 4. Section 5 contains details on the experiments that were performed to test our algorithm. Finally, conclusions and future research directions are given in Sect. 6.

\section{GRASP+VND and GRASP+VNS for the TRP}

As mentioned in the introduction, our method for the TRP combines a GRASP construction phase with a VND or a VNS improvement phase. Both phases are repeated a number of times, and the best solution found is reported. An outline of the algorithm is shown in Algorithm 1.

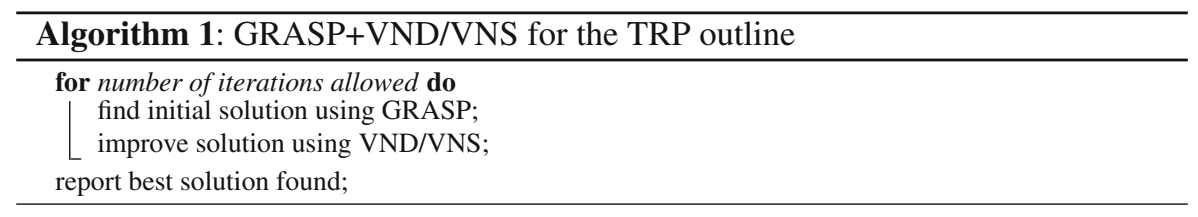

\subsection{Solution representation and objective function calculation}

A solution in our algorithm is represented as a permutation of the vertices from $v_{1}$ to $v_{n}$. From this representation, the objective function value can be easily calculated. Assume a solution $x=x_{1}, x_{2}, \ldots, x_{n}$ with $x_{1}$ being the first vertex in the solution, $x_{2}$ the second, and so on. The total latency for this solution can be calculated as

$$
f(x)=\sum_{i=1}^{n}(n-i+1) w\left(x_{i-1}, x_{i}\right)
$$

keeping in mind that $x_{0} \equiv v_{0}$. This calculation is equivalent to the more intuitive calculation in which the latencies of the vertices in the solution are summed:

$$
f(x)=\sum_{i=1}^{n} l\left(x_{i}\right) \quad \text { where } \quad l\left(x_{i}\right)=\sum_{j=1}^{i} w\left(x_{j-1}, x_{j}\right) .
$$

Equation 1 is computationally more efficient when e.g., vertices are added or deleted.

In Fig. 2, we show an example solution for the TRP in Fig. 1. The objective function of this solution is calculated as

$$
f(x)=6(2.1)+5(6.3)+4(4.0)+3(1.8)+2(5.2)+1(2.2)=78.1 .
$$


Fig. 2 Example traveling repairman problem solution

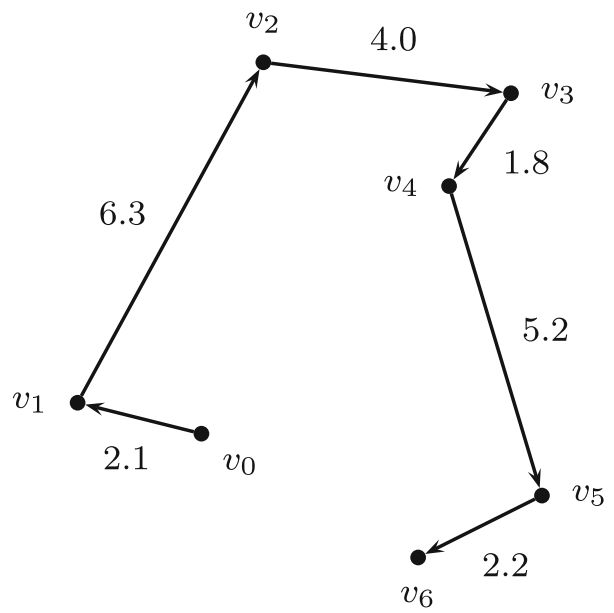

Fig. 3 Proximity matrix for the example in Fig. 1

\begin{tabular}{c|cccccc} 
& 1st & 2 nd & 3 rd & 4 rd & 5 th & 6 th \\
\hline$v_{0}$ & $v_{1}$ & $v_{6}$ & $v_{5}$ & $v_{4}$ & $v_{2}$ & $v_{3}$ \\
$v_{1}$ & $v_{0}$ & $v_{6}$ & $v_{2}$ & $v_{4}$ & $v_{5}$ & $v_{3}$ \\
$v_{2}$ & $v_{4}$ & $v_{3}$ & $v_{0}$ & $v_{1}$ & $v_{5}$ & $v_{6}$ \\
$v_{3}$ & $v_{4}$ & $v_{2}$ & $v_{5}$ & $v_{0}$ & $v_{6}$ & $v_{1}$ \\
$v_{4}$ & $v_{3}$ & $v_{2}$ & $v_{5}$ & $v_{0}$ & $v_{6}$ & $v_{1}$ \\
$v_{5}$ & $v_{6}$ & $v_{4}$ & $v_{0}$ & $v_{3}$ & $v_{1}$ & $v_{2}$ \\
$v_{6}$ & $v_{5}$ & $v_{0}$ & $v_{4}$ & $v_{1}$ & $v_{3}$ & $v_{2}$
\end{tabular}

Many of the heuristics developed in the following sections require finding the closest, second-closest, etc. vertex to any given vertex. We therefore pre-process the distance data and maintain in memory a proximity matrix that contains one row per vertex. The $(i+1)$-th row of the matrix contains all vertices (except for vertex $v_{i}$ ) in order of proximity to vertex $v_{i}$. For the example in Fig. 1, the proximity matrix is shown in Fig. 3.

\subsection{GRASP}

The motivation for using GRASP as a procedure for the TRP is the observation that the initial choices of vertices to visit, i.e., the first few vertices in the tour, are more important than later ones. This immediately follows from Eq. 1, from which it is clear that the $i$-th distance in the solution is multiplied with a factor $n-i+1$. We therefore expect a greedy algorithm to perform well, as it attempts to add the shortest possible distances early on and leaves the longer ones for later. A completely greedy algorithm on the other hand, can be expected to miss some interesting opportunities.

Perhaps the most distinguishing characteristic of GRASP is the way in which it combines greediness with randomness in the construction phase. To this end, a restricted candidate list (RCL) is built by selecting a subset of all elements in a greedy fashion. Assuming a minimization problem, the RCL contains the elements whose incorporation into the partially built solution would yield the smallest increase in objective 
function value. From the RCL, an element is then selected at random, after which the RCL is updated to reflect the fact that a new element was added to the solution and is no longer available for selection. Selection of an element and update of the RCL are repeated until a complete solution has been built. From this solution, a local search phase starts until a local optimum is found. The size of the RCL, $\alpha$, is a parameter of the GRASP algorithm that controls the balance between greediness and randomness. If $\alpha$ is small, the search is relatively greedy. If $\alpha$ is large, it is relatively random. In the extreme cases, setting $\alpha=1$ leads to a completely deterministic greedy search, whereas setting $\alpha=n$ entails a completely random search.

The RCL concept can be easily translated to the TRP problem, and efficiently implemented using the proximity matrix (see Fig. 3). Starting from vertex $v_{0}$, the RCL is filled with the $\alpha$ closest vertices. From this RCL, a random vertex is chosen, say $v_{r}$. Now, the RCL is filled with the $\alpha$ vertices closest to $v_{r}$, omitting any elements that have already been selected. If fewer than $\alpha$ vertices remain, then the size of the RCL is decreased. A pseudo-code version of the GRASP construction phase of our algorithm for the TRP is in Algorithm 2.

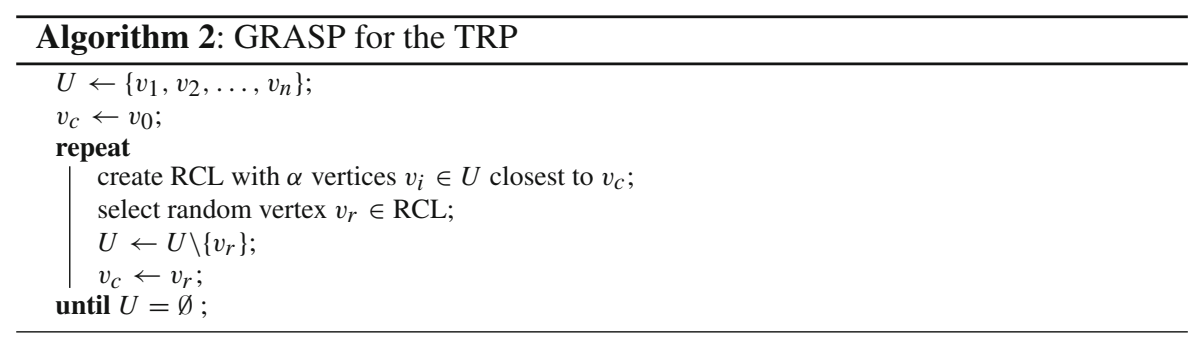

2.3 Variable neighborhood descent and variable neighborhood search

As their names suggest, these metaheuristics systematically explore different neighborhood structures. The main idea underlying VNS and VND is that a local optimum relative to a certain neighborhood structure not necessarily is a local optimum relative to another neighborhood structure. For this reason, escaping from a local optimum can be done by changing the neighborhood structure.

Although this is not required, many implementations of VNS and VND use a sequence of nested neighborhoods, $\mathcal{N}_{1}$ to $\mathcal{N}_{k_{\max }}$, in which each neighborhood in the sequence is a superset of its predecessor, i.e., $\mathcal{N}_{k} \subset \mathcal{N}_{k+1}$. The neighborhoods we use do not possess this property.

Pseudo-code for a basic version of VND is given in Algorithm 3.

The difference between VNS and VND is that the former uses a so-called perturbation, also called shaking, move. The aim of the perturbation move is to disturb the current solution in such a way that its structure is partially (but not completely) destroyed. Usually, the perturbation move is executed before the set of neighborhoods is used to improve the solution. In our algorithm, the perturbation is performed before 


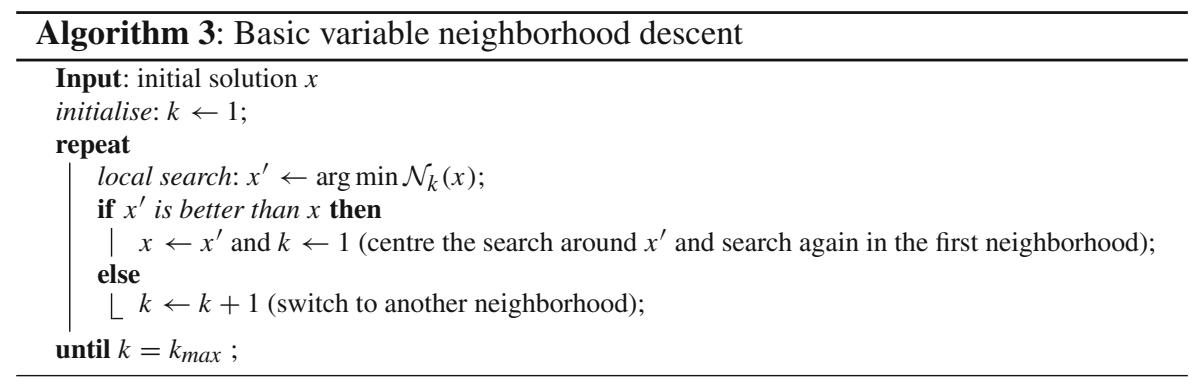

each neighborhood, since we found that this yielded better results. The perturbation move of our VNS algorithm randomly removes a certain fixed percentage $(20 \%$ in our experiments) of the customers in the current solution and inserts them in a different, random location. Pseudo-code for the variant of VNS that is used in our algorithm is given in Algorithm 4. Our VNS deviates from the "standard" VNS in that it does not re-center around the best-known solution after the perturbation phase.

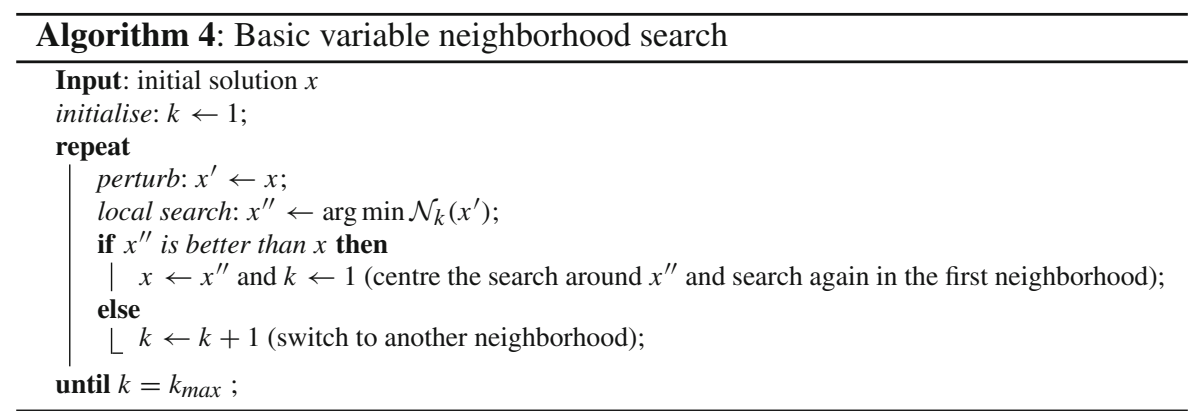

Usually, VNS/VND algorithms examine all neighborhoods in a certain order, from small to large. The reason is that small neighborhoods contain fewer solutions and can hence be searched in less time than large neighborhoods. Larger neighborhoods are only examined when all smaller neighborhoods have been depleted, i.e., when the current solution is a local optimum relative to all smaller neighborhoods. The VND stops when the solution is a local optimum of all neighborhoods. Another option is to explore the neighborhoods in the order of their effectiveness with respect to the problem at hand, which can be established by means of a small pilot study.

\subsubsection{Neighborhoods}

Our search uses five neighborhood structures: swap, swap-adjacent, 2-opt, Or-opt, and remove-insert. In a pilot study, we found that the performance of the algorithm is relatively insensitive to the order in which the neighborhoods are used. The neighborhoods 
are therefore explored in a specific order, from "small" to "large" as it is common in VNS algorithms, i.e., swap-adjacent, remove-insert, swap, 2-opt and Or-opt.

The swap heuristic attempts to swap the positions of each pair of vertices in the solution (see Fig. 4). Searching the entire neighborhood of a solution with this heuristic requires quadratic time in the problem size $\left(O\left(n^{2}\right)\right)$. Using Eq. 1, examining the effect of a swap of vertices $x_{i}$ and $x_{j}$ in solution $x$ on the objective function value can be easily done by subtracting the weight of the removed edges and adding that of the new edges in the solution.

The swap-adjacent heuristic, as its name suggests, attempts to swap each pair of adjacent vertices in the solution $x$. This heuristic examines a subset of the moves explored in the swap heuristic and has a linear complexity $(O(n))$.

The 2-opt heuristic (see Fig. 5) removes each pair of edges from the solution and reconnects the vertices. Updating the objective function is more complicated for 2-opt, since the middle part of the solution (that was "cut out") is reversed. This heuristic requires $O\left(n^{2}\right)$ time to examine the entire neighborhood of a solution.

The Or-opt heuristic (see Fig. 6) removes each triplet of edges from the solution and reconnects the vertices in such a way that the orientation of the solution parts is preserved. The time complexity of this heuristic is cubic $\left(O\left(n^{3}\right)\right)$.

The remove-insert heuristic examines each vertex $x_{i}$ in the solution and places the vertex furthest away from $x_{i}$ at the end of the solution. This heuristic has linear complexity like the swap-adjacent heuristic.
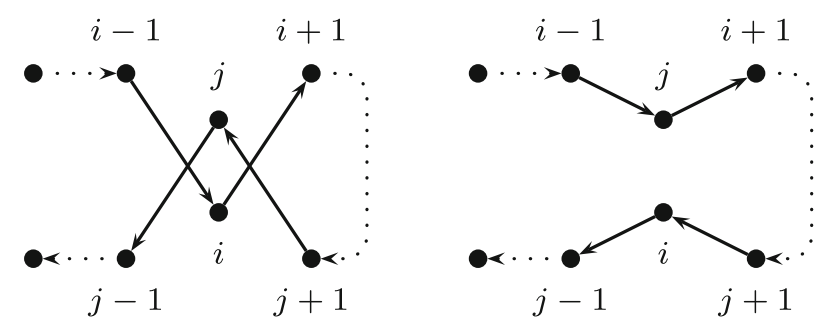

Fig. 4 Swap
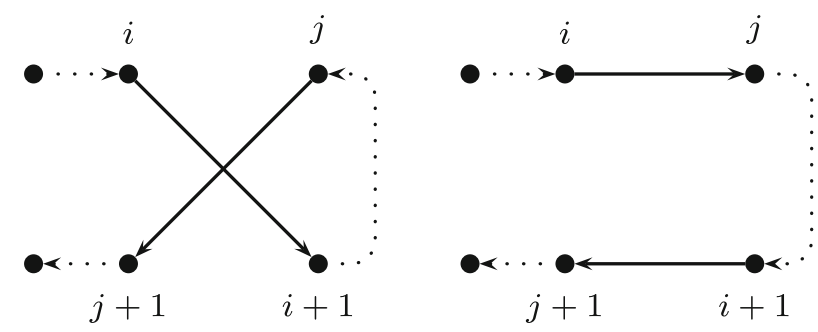

Fig. 5 2-opt 

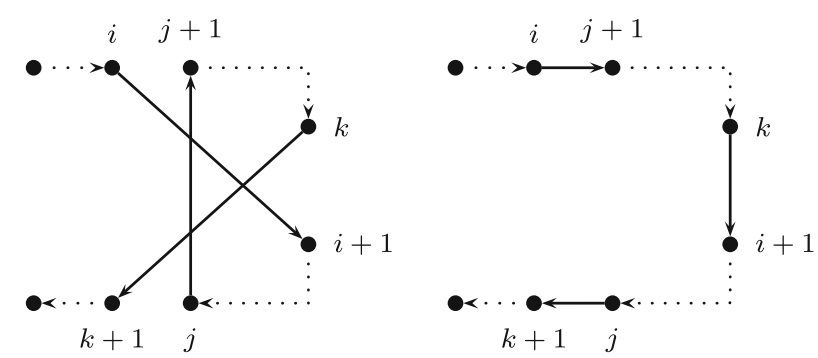

Fig. 6 Or-opt

\subsubsection{Neighborhood reduction}

We made several attempts to decrease the size of the neighborhoods to examine during the local search phase. All neighborhood reduction schemes are carefully tested in Sect. 5.

For the swap, 2-opt and Or-opt moves the neighborhood size is reduced by fixing a random vertex and then attempting only the moves that involve this vertex. We call this strategy "Fix" and compare it to the "NoFix" strategy in which the entire neighborhood is searched without first fixing a random vertex. This neighborhood reduction scheme decreases the size of the neighborhood by a factor $O(n)$.

A second neighborhood reduction scheme is based on the observation that in a reasonably good solution, most improving moves will involve vertices that appear in relative proximity in this solution. For example, in a good solution it is unlikely that a swap will yield a better solution if it exchanges a vertex that appears close to the depot with one that appears far from the depot. We therefore introduce a proximity factor $\beta$ to determine the maximum distance between vertices that may be involved in a move. The factor $\beta$ has a slightly different influence, depending on the move. For a swap move, the second vertex may be only $\beta$ positions further in the solution than the first one. For a 2-opt move, the head of the second chosen arc may only be $\beta$ positions further in the solution than the head of the first chosen arc. For an Or-opt move, the heads of both the second and third chosen arcs may be only $\beta$ positions away from the head of the first arc. For the swap-adjacent and remove-insert heuristics, we did not use this strategy.

\section{Bounds for the TRP}

We compare the outcome of our algorithm to an upper and a lower bound, discussed in this section. Due to the specific properties of the TRP, deriving good bounds is difficult. As explained in Blum et al. (1994), the TRP cannot be simply considered a variant of the TSP. The gap between the upper and the lower bounds are therefore relatively large. 


\subsection{Upper bound}

Like Simchi-Levi and Berman (1991) and Bianco et al. (1993), we use the nearest neighbor heuristic to derive an upper bound for the TRP. The nearest neighborhood heuristic belongs to the family of greedy algorithms and constructs an initial solution by starting from the depot $\left(v_{0}\right)$ and repeatedly adding the closest vertex to the last-added one until all vertices have been added. It is a special case of our GRASP procedure, in which the RCL only contains the closest vertex not yet added to the solution, i.e., in which $\alpha=1$.

As an example, application of the nearest neighbor heuristic to the example in Fig. 1 (left) yields the solution $v_{1} v_{6} v_{5} v_{4} v_{3} v_{2}$ as shown in Fig. 7. Next to each edge, the multiplication factor is shown. The upper bound for this example therefore has a value of

$$
\mathrm{UB}=6(2.1)+5(6.0)+4(2.2)+3(5.2)+2(1.8)+1(4.0)=74.6 .
$$

\subsection{Lower bound}

Our lower bound for the TRP is a variant of the minimum spanning tree (MST) lower bound for the TSP. It is computed by sorting the edges of the MST of the graph in order of increasing weight and multiplying each edge with a factor like the edges of the TRP solution in Eq. 1. The smallest edge is multiplied with $n$, the second-smallest with $n-1$, etc. The largest edge is multiplied with a factor 1 .

The lower bound for the example in Fig. 1 is shown in Fig. 7 (right). The value of the lower bound is

$$
\mathrm{LB}=6(1.8)+5(2.1)+4(2.2)+3(3.6)+2(4.0)+1(5.2)=54.1 .
$$

Proposition 1 Let $t=t_{1}, t_{2}, \ldots, t_{n}, t_{i} \in E$, be the edges of the minimum spanning tree of the graph $G=(V, E, w)$, sorted in increasing order of weight, i.e., $w\left(t_{i}\right) \leq w\left(t_{j}\right)$ iff $i<j$. A lower bound for the TRP on $G$ is given by
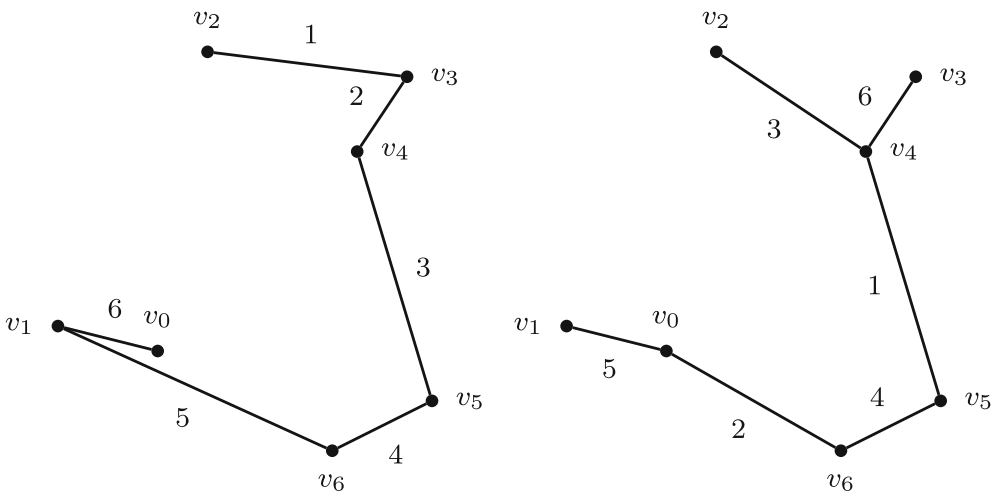

Fig. 7 Upper bound (left) and lower bound (right) for the problem in Fig. 1 


$$
L B=\sum_{i=1}^{n}(n-i+1) w\left(t_{i}\right)
$$

where $w\left(t_{i}\right)$ is the weight of edge $t_{i}$.

Proof Assume that the optimal traveling repairman solution of $G=(V, E, w)$ is given by $r=r_{1}, r_{2}, \ldots, r_{n}, r_{i} \in E$, with the edges in order of appearance in the solution. The objective function value of $r$ is $f(r)=\sum_{i=1}^{n}(n-i+1) w\left(r_{i}\right)$.

Since $t$ is a minimum spanning tree, the edges $t_{1}, t_{2}, \ldots, t_{i}$ form the set of the $i$ smallest edges of the graph that do not form a cycle. It holds that the sum of the weights of the $i$ smallest edges that do not form a cycle, is smaller than the sum of the weights of any other $i$ edges that do not form a cycle. Since the first $i$ edges in the traveling repairman solution also do not form a cycle, it follows that

$$
\sum_{j=1}^{i} w\left(t_{j}\right) \leq \sum_{j=1}^{i} w\left(r_{j}\right)
$$

Summing over $i$, we get that

$$
\sum_{i=1}^{n} \sum_{j=1}^{i} w\left(t_{j}\right) \leq \sum_{i=1}^{n} \sum_{j=1}^{i} w\left(r_{j}\right)
$$

which simplifies to

$$
\sum_{i=1}^{n}(n-i+1) w\left(t_{i}\right) \leq \sum_{i=1}^{n}(n-i+1) w\left(r_{i}\right) .
$$

\section{Benchmark instance set generation}

To the best of our knowledge, no standard set of medium- and large-scale benchmark instances for the TRP exists. We therefore test our algorithm on (1) an extensive set of 140 self-generated symmetric instances of seven different problem sizes ranging from 10 to 1000 vertices $(10,20,50,100,200,500,1000)$ and (2) a selection of ten instances from the TSP Library.

To create our own set of 140 test instances, we have generated 20 random instances for each problem size. For each instance, vertex coordinates have been generated from a uniform distribution between 0 and 100 (or between 0 and 500 for the instances of size 500 and 1000). All distances are Euclidean, rounded down to the nearest integer. The instances are available from the authors upon request (Table. 1). 
Table 1 Random instances for the TRP

\begin{tabular}{lrc}
\hline File name & Size & {$[$ min,max $]$} \\
\hline TRP-S10-Rx & 10 & $(0,100)$ \\
TRP-S20-Rx & 20 & $(0,100)$ \\
TRP-S50-Rx & 50 & $(0,100)$ \\
TRP-S100-Rx & 100 & $(0,100)$ \\
TRP-S200-Rx & 200 & $(0,100)$ \\
TRP-S500-Rx & 500 & $(0,500)$ \\
TRP-S1000-Rx & 1000 & $(0,500)$ \\
\hline
\end{tabular}

\section{Experiments}

\subsection{General experiment setup}

Several parameters need to be set for our algorithms. Concerning the construction phase, a pilot study clearly showed that the greediness of the GRASP method needs to be large, i.e., values of $\alpha>2$ only very rarely yielded better results than those found for the same problem with $\alpha \leq 2$. As pointed out above, the importance of adding the smallest edges in the early stages of a construction procedure is not surprising given their importance in the objective function. We therefore test our procedure with $\alpha=2$.

The first neighborhood reduction scheme is tested by running the algorithms with both implementations of the neighborhoods. Runs in which the search starts by selecting a fixed random vertex are labeled "Fix". Runs in which the search explores all possible moves are labeled "Nofix".

The implementation of the second neighborhood reduction scheme depends on the size of the problem. For problems of size $n<500$, a "Full" strategy is tested with proximity factor $\beta=n$ against a "Half" strategy with $\beta=n / 2$. For problems with 500 and 1000 vertices, $\beta$ equals 250 in the Full strategy for swap, 2-opt and Or-opt. In the Half strategy, $\beta$ equals 300, 200 and 100 for swap, 2-opt and Or-opt respectively. The settings of $\beta$ were obtained using a small pilot study. For problems of size 10 , the Half strategy is ignored.

We also compare a multi-start version of our algorithms in which 10 solutions are generated with the GRASP procedure using $\alpha=2$, each of which is improved using VNS or VND. The best solution found by these 10 independent runs is reported.

The two neighborhood reduction schemes are combined with the single- and multistart setting, giving rise to eight different settings of our algorithms. These eight settings are shown in Table 2.

Finally, both the GRASP+VND and the GRASP+VNS algorithms are tested on the same problem instances, with the same parameters. For the VNS routine, the percentage of vertices to move in the perturbation move was set at $20 \%$.

All the computations reported in this section have been carried out on a Personal Computer with Pentium 4, $2.4 \mathrm{GHz}$ processor and $512 \mathrm{MB}$ RAM. The algorithm was coded in $\mathrm{C}++$. 
Table 2 Experiment settings for GRASP+VNS and GRASP+VND

Table 3 Comparison of the GRASP+VNS metaheuristic with CPLEX on instances with 10 customers

Best result in bold

\begin{tabular}{llll}
\hline Restarts & $\begin{array}{l}\text { Neighborhood } \\
\text { implementation }\end{array}$ & $\begin{array}{l}\text { Proximity } \\
\text { rule }\end{array}$ & $\begin{array}{l}\text { Experiment } \\
\text { number }\end{array}$ \\
\hline Single-start & Nofix & Full & 1 \\
& Fix & Half & 2 \\
& & Full & 3 \\
Multi-start & Nofix & Half & 4 \\
& & Full & 5 \\
& Fix & Half & 6 \\
& & Full & 7 \\
& & Half & 8 \\
\hline
\end{tabular}

\begin{tabular}{|c|c|c|c|c|}
\hline & \multicolumn{2}{|c|}{ GRASP+VNS } & \multicolumn{2}{|c|}{ CPLEX } \\
\hline & Obj. & $T$ & Obj. & $T$ \\
\hline $\mathrm{R} 1$ & 1303 & 0.00 & 1303 & 81 \\
\hline $\mathrm{R} 2$ & 1517 & 0.00 & 1517 & 61 \\
\hline $\mathrm{R} 3$ & 1233 & 0.00 & 1233 & 65 \\
\hline $\mathrm{R} 4$ & 1386 & 0.00 & 1386 & 90 \\
\hline R5 & 978 & 0.00 & 978 & 61 \\
\hline R6 & 1477 & 0.00 & 1477 & 217 \\
\hline R7 & 1163 & 0.00 & 1163 & 146 \\
\hline $\mathrm{R} 8$ & 1234 & 0.00 & 1234 & 222 \\
\hline R9 & 1402 & 0.00 & 1402 & 164 \\
\hline $\mathrm{R} 10$ & 1388 & 0.00 & 1388 & 261 \\
\hline $\mathrm{R} 11$ & 1405 & 0.00 & 1405 & 122 \\
\hline $\mathrm{R} 12$ & 1150 & 0.00 & 1150 & 125 \\
\hline $\mathrm{R} 13$ & 1531 & 0.00 & 1531 & 570 \\
\hline $\mathrm{R} 14$ & 1219 & 0.00 & 1219 & 122 \\
\hline $\mathrm{R} 15$ & 1087 & 0.00 & 1087 & 127 \\
\hline $\mathrm{R} 16$ & 1264 & 0.00 & 1264 & 489 \\
\hline $\mathrm{R} 17$ & 1058 & 0.00 & 1058 & 30 \\
\hline $\mathrm{R} 18$ & 1083 & 0.00 & 1083 & 34 \\
\hline R19 & 1394 & 0.00 & 1394 & 228 \\
\hline $\mathrm{R} 20$ & 951 & 0.00 & 951 & 25 \\
\hline Average & & 0.00 & & 162 \\
\hline
\end{tabular}

\subsection{Comparison with CPLEX on small instances}

A comparison of our GRASP+VNS with CPLEX on small instances is reported in Tables 3 and 4. In each of these cases, we ran the best two configurations of our metaheuristic (multi-start GRASP+VNS with Fix neighborhood implementation and Half or Full proximity rule) on all the instances and report the best of the two results. Table 3 displays the results of both methods on instances with 10 customers. Column 
Table 4 Comparison of the GRASP+VNS metaheuristic with CPLEX on instances with 20 customers

\begin{tabular}{|c|c|c|c|c|c|c|c|c|}
\hline & \multicolumn{2}{|c|}{ GRASP+VNS } & \multicolumn{3}{|c|}{ CPLEX (1h) } & \multicolumn{3}{|c|}{ CPLEX (24h) } \\
\hline & Obj. & $T$ & Obj. & Gap (\%) & $\Delta(\%)$ & Obj. & Gap (\%) & $\Delta(\%)$ \\
\hline $\mathrm{R} 1$ & 3175 & $<0.02$ & 3224 & 41.55 & 1.52 & 3175 & 22.17 & 0.00 \\
\hline $\mathrm{R} 2$ & 3248 & $<0.02$ & 3440 & 57.30 & 5.58 & 3248 & 23.56 & 0.00 \\
\hline $\mathrm{R} 3$ & 3570 & $<0.02$ & 4141 & 57.48 & 13.79 & 3570 & 31.09 & 0.00 \\
\hline $\mathrm{R} 4$ & 2983 & $<0.02$ & 3317 & 48.51 & 10.07 & 3065 & 17.81 & 2.68 \\
\hline R5 & 3248 & $<0.02$ & 3732 & 59.28 & 12.97 & 3365 & 39.22 & 3.48 \\
\hline R6 & 3328 & $<0.02$ & 3426 & 55.96 & 2.86 & 3328 & 33.11 & 0.00 \\
\hline R7 & 2809 & $<0.02$ & 3286 & 61.23 & 14.52 & 2809 & 41.07 & 0.00 \\
\hline $\mathrm{R} 8$ & 3461 & $<0.02$ & 3605 & 54.37 & 3.99 & 3461 & 22.14 & 0.00 \\
\hline R9 & 3475 & $<0.02$ & 4285 & 58.87 & 18.90 & 3392 & 19.33 & -2.45 \\
\hline R10 & 3359 & $<0.02$ & 3707 & 56.31 & 9.39 & 3359 & 21.25 & 0.00 \\
\hline R11 & 2916 & $<0.02$ & 3074 & 49.60 & 5.14 & 2916 & 26.64 & 0.00 \\
\hline $\mathrm{R} 12$ & 3314 & $<0.02$ & 3468 & 47.11 & 4.44 & 3314 & 21.11 & 0.00 \\
\hline $\mathrm{R} 13$ & 3412 & $<0.02$ & 3427 & 56.58 & 0.44 & 3412 & 27.78 & 0.00 \\
\hline R14 & 3297 & $<0.02$ & 3458 & 56.59 & 4.66 & 3297 & 23.45 & 0.00 \\
\hline R15 & 2862 & $<0.02$ & 2881 & 39.88 & 0.66 & 2862 & 19.12 & 0.00 \\
\hline R16 & 3433 & $<0.02$ & 3909 & 57.01 & 12.18 & 3433 & 23.22 & 0.00 \\
\hline R17 & 2924 & $<0.02$ & 2977 & 57.47 & 1.78 & 2924 & 21.15 & 0.00 \\
\hline R18 & 3168 & $<0.02$ & 3467 & 61.08 & 8.62 & 3150 & 31.03 & -0.57 \\
\hline R19 & 3299 & $<0.02$ & 3503 & 42.35 & 5.82 & 3299 & 19.87 & 0.00 \\
\hline R20 & 2796 & $<0.02$ & 2842 & 42.18 & 1.62 & 2796 & 15.11 & 0.00 \\
\hline Average & & $<0.02$ & & 53.04 & 6.95 & & 25.06 & 0.16 \\
\hline
\end{tabular}

Best result in bold

$T$ shows the computing time in seconds. As can be seen, CPLEX finds the optimal solutions to all 20 random instances in an average time of 162 seconds. Our method finds all optimal solutions as well, albeit in a much smaller amount of time (less than 0.005 seconds) in each case.

In Table 4, results for instances with 20 customers are shown. In this case, CPLEX is not able to prove the optimality of the solutions it finds in 1 day of computing time: the average optimality gap is $53 \%$ after 1 hour and $25 \%$ after 24 hours of computation. In 16 out of 20 instances our GRASP+VNS metaheuristic finds the same solution as CPLEX (in less than 0.02 seconds for each case), and in 2 cases (R4 and R5) it finds a better solution. In only two cases is CPLEX able to find a better solution after 24 hours of calculation. Gaps for larger instances were considered too large to provide a meaningful benchmark. These findings are in line with those of Méndez-Díaz et al. (2008), who conclude that the TRP is a very hard problem to solve to optimality.

\subsection{Results on larger instances}

The full results of our GRASP+VND algorithm on the randomly generated data sets are reported in Table 5, while those of the GRASP+VNS algorithm are in Table 6. 
Table 5 Results of the GRASP+VND

\begin{tabular}{|c|c|c|c|c|c|c|c|c|}
\hline \multirow[t]{3}{*}{$n$} & \multicolumn{8}{|c|}{ Single-start } \\
\hline & \multicolumn{4}{|c|}{1 (Nofix-Full) } & \multicolumn{4}{|c|}{2 (Nofix-Half) } \\
\hline & $\mathrm{UB} \%$ & $\mathrm{LB} \%$ & $T$ & $T_{\text {best }}$ & $\mathrm{UB} \%$ & $\mathrm{LB} \%$ & $T$ & $T_{\text {best }}$ \\
\hline 10 & 2.14 & 33.48 & 0.00 & 0.00 & - & - & - & - \\
\hline 20 & 8.19 & 42.43 & 0.00 & 0.00 & 6.89 & 44.75 & 0.00 & 0.00 \\
\hline 50 & 6.85 & 50.07 & 0.27 & 0.21 & 6.70 & 50.21 & 0.03 & 0.00 \\
\hline 100 & 10.24 & 45.53 & 9.73 & 8.91 & 9.99 & 45.97 & 1.21 & 1.11 \\
\hline 200 & 10.69 & 43.37 & 356.73 & 319.45 & 10.20 & 44.18 & 39.81 & 35.02 \\
\hline 500 & 10.70 & 46.49 & 7192.94 & 7002.09 & 10.49 & 46.84 & 635.96 & 598.00 \\
\hline 1000 & - & - & - & - & 11.32 & 46.40 & 3378.28 & 3013.29 \\
\hline \multirow[t]{3}{*}{$n$} & \multicolumn{8}{|c|}{ Single-start } \\
\hline & \multicolumn{4}{|c|}{3 (Fix-Full) } & \multicolumn{4}{|c|}{4 (Fix-Half) } \\
\hline & $\mathrm{UB} \%$ & $\mathrm{LB} \%$ & $T$ & $T_{\text {best }}$ & $\mathrm{UB} \%$ & $\mathrm{LB} \%$ & $T$ & $T_{\text {best }}$ \\
\hline 10 & 1.96 & 33.71 & 0.00 & 0.00 & - & - & - & - \\
\hline 20 & 7.51 & 43.45 & 0.00 & 0.00 & 6.51 & 45.19 & 0.00 & 0.00 \\
\hline 50 & 5.69 & 51.95 & 0.01 & 0.00 & 5.24 & 52.64 & 0.00 & 0.00 \\
\hline 100 & 8.55 & 48.33 & 0.32 & 0.11 & 8.56 & 48.30 & 0.07 & 0.04 \\
\hline 200 & 8.61 & 46.77 & 6.30 & 4.98 & 8.54 & 46.88 & 1.25 & 1.09 \\
\hline 500 & 9.19 & 48.97 & 134.83 & 112.14 & 9.18 & 49.00 & 18.86 & 13.30 \\
\hline 1000 & 9.31 & 49.72 & 837.26 & 787.63 & 9.22 & 49.88 & 112.99 & 106.37 \\
\hline \multirow[t]{3}{*}{$n$} & \multicolumn{8}{|c|}{ Multi-start } \\
\hline & \multicolumn{4}{|c|}{5 (Nofix-Full) } & \multicolumn{4}{|c|}{6 (Nofix-Half) } \\
\hline & $\mathrm{UB} \%$ & $\mathrm{LB} \%$ & $T$ & $T_{\text {best }}$ & $\mathrm{UB} \%$ & $\mathrm{LB} \%$ & $T$ & $T_{\text {best }}$ \\
\hline 10 & 2.44 & 33.04 & 0.00 & 0.00 & - & - & - & - \\
\hline 20 & 9.86 & 39.63 & 0.04 & 0.00 & 9.80 & 39.80 & 0.00 & 0.00 \\
\hline 50 & 9.67 & 45.41 & 3.21 & 3.01 & 9.48 & 45.71 & 0.43 & 0.31 \\
\hline 100 & 11.56 & 43.40 & 101.27 & 91.12 & 11.21 & 43.93 & 12.55 & 10.79 \\
\hline 200 & 11.33 & 42.32 & 3741.05 & 3225.03 & 11.44 & 42.14 & 444.43 & 397.42 \\
\hline 500 & 8.11 & 50.73 & 91470.78 & 86431.76 & 11.47 & 45.22 & 6335.79 & 6115.69 \\
\hline 1000 & - & - & - & - & 8.18 & 51.57 & 9114.75 & 8912.23 \\
\hline \multirow[t]{3}{*}{$n$} & \multicolumn{8}{|c|}{ Multi-start } \\
\hline & \multicolumn{4}{|c|}{7 (Fix-Full) } & \multicolumn{4}{|c|}{8 (Fix-Half) } \\
\hline & $\mathrm{UB} \%$ & $\mathrm{LB} \%$ & $T$ & $T_{\text {best }}$ & $\mathrm{UB} \%$ & $\mathrm{LB} \%$ & $T$ & $T_{\text {best }}$ \\
\hline 10 & 2.44 & 33.04 & 0.00 & 0.00 & - & - & - & - \\
\hline 20 & 9.41 & 40.34 & 0.01 & 0.00 & 9.70 & 39.82 & 0.00 & 0.00 \\
\hline 50 & 8.29 & 47.59 & 0.19 & 0.10 & 7.83 & 48.40 & 0.04 & 0.00 \\
\hline
\end{tabular}


Table 5 continued

\begin{tabular}{|c|c|c|c|c|c|c|c|c|}
\hline \multirow[t]{3}{*}{$n$} & \multicolumn{8}{|c|}{ Multi-start } \\
\hline & \multicolumn{4}{|c|}{7 (Fix-Full) } & \multicolumn{4}{|c|}{8 (Fix-Half) } \\
\hline & $\mathrm{UB} \%$ & $\mathrm{LB} \%$ & $T$ & $T_{\text {best }}$ & $\mathrm{UB} \%$ & $\mathrm{LB} \%$ & $T$ & $T_{\text {best }}$ \\
\hline 100 & 8.43 & 48.43 & 3.54 & 3.01 & 8.80 & 47.82 & 0.80 & 0.62 \\
\hline 200 & 7.75 & 48.07 & 78.25 & 61.22 & 7.76 & 48.05 & 16.56 & 13.71 \\
\hline 500 & 8.11 & 50.73 & 1534.90 & 1418.73 & 8.07 & 50.81 & 239.44 & 216.41 \\
\hline 1000 & - & - & - & - & 8.09 & 51.73 & 1431.16 & 1297.09 \\
\hline
\end{tabular}

The results of the GRASP+VND algorithm on the TSP Library instances can be found in Tables 7, 8 and 9. In all tables, the column labeled UB\% shows the improvement of the objective function over the upper bound obtained with the nearest neighbor heuristic. The column labeled LB\% shows the gap to the lower bound, obtained using the minimum spanning tree with edge multiplicator. In the column labeled $T$, the computation time until the algorithm stops (in seconds) is shown. Column $T_{\text {best }}$ in Tables 5 and 6 contains the computation time (in seconds) at which the best solution was reached by the GRASP+VND and GRASP+VNS algorithms respectively.

From Tables 5 to 6 we can draw some conclusions about the working of our algorithm. Unsurprisingly, the multi-start version of our algorithm (algorithm settings 5 to 8) requires a much larger computation time than the single-start version (settings 1 to 4). However, the quality improvement obtained by this method is relatively small. The perturbations in the GRASP+VNS algorithm (Table 6) seem to help marginally, as the solutions obtained by this algorithm are usually slightly better. This may indicate that the GRASP multi-start is not able to provide enough diversification, and that the perturbation move is useful.

Both neighborhood reduction strategies seem to work well. The neighborhood implementation with fixed random vertex (Fix, settings 3, 4, 7, and 8) uses significantly less computing time, combined with a rather small loss of solution quality. The Half neighborhood reduction strategy (settings 2, 4, 6, and 8) yields a much faster solution method, at the cost of some loss in objective function quality. Both neighborhood reduction strategies prove useful for the largest instances (500 and 1000 vertices), for which full neighborhood search (Full and NoFix) was too time consuming.

Tables 7, 8 and 9 show the results of the algorithm on a selected set of TSPlib instances. Conclusions on the working of our algorithm that can be drawn from these experiments are in line with the ones on randomly generated instances. Table 9 showsunsurprisingly - that the optimal TSP-solution is generally not a good solution to the TRP on the same instance. On average, the best solution found by our algorithm is almost $17 \%$ better than the optimal TSP-solution.

\section{Conclusions and future research}

In this paper, we have presented the first metaheuristics for the traveling repairman problem. These metaheuristics consists of a GRASP construction phase and a variable 
Table 6 Results of the GRASP+VNS

\begin{tabular}{|c|c|c|c|c|c|c|c|c|}
\hline \multirow[t]{3}{*}{$n$} & \multicolumn{8}{|c|}{ Single-start } \\
\hline & \multicolumn{4}{|c|}{1 (Nofix-Full) } & \multicolumn{4}{|c|}{2 (Nofix-Half) } \\
\hline & $\mathrm{UB} \%$ & $\mathrm{LB} \%$ & $T$ & $T_{\text {best }}$ & $\mathrm{UB} \%$ & $\mathrm{LB} \%$ & $T$ & $T_{\text {best }}$ \\
\hline 10 & 2.14 & 33.48 & 0.00 & 0.00 & - & - & - & - \\
\hline 20 & 8.19 & 42.43 & 0.00 & 0.00 & 6.89 & 44.75 & 0.00 & 0.00 \\
\hline 50 & 6.87 & 50.05 & 0.29 & 0.18 & 6.69 & 50.21 & 0.03 & 0.00 \\
\hline 100 & 10.54 & 45.04 & 11.99 & 8.32 & 10.33 & 45.42 & 1.68 & 1.11 \\
\hline 200 & 10.82 & 43.16 & 378.68 & 311.02 & 10.18 & 44.23 & 46.11 & 3.75 \\
\hline 500 & 10.93 & 46.12 & 7373.09 & 6951.22 & 10.93 & 46.11 & 693.16 & 604.89 \\
\hline 1000 & - & - & - & - & 11.57 & 45.99 & 4103.28 & 3876.51 \\
\hline
\end{tabular}

\begin{tabular}{|c|c|c|c|c|c|c|c|c|}
\hline \multirow[t]{3}{*}{$n$} & \multicolumn{8}{|c|}{ Single-start } \\
\hline & \multicolumn{4}{|c|}{3 (Fix-Full) } & \multicolumn{4}{|c|}{4 (Fix-Half) } \\
\hline & UB\% & LB\% & $T$ & $T_{\text {best }}$ & $\mathrm{UB} \%$ & LB\% & $T$ & $T_{\text {best }}$ \\
\hline$\overline{10}$ & 1.96 & 33.71 & 0.00 & 0.00 & - & - & - & - \\
\hline 20 & 7.51 & 43.45 & 0.00 & 0.00 & 6.51 & 45.19 & 0.00 & 0.00 \\
\hline 50 & 5.69 & 51.94 & 0.02 & 0.00 & 5.27 & 52.60 & 0.00 & 0.00 \\
\hline 100 & 9.04 & 47.53 & 0.36 & 0.21 & 8.73 & 48.02 & 0.09 & 0.06 \\
\hline 200 & 13.40 & 38.87 & 7.57 & 5.99 & 9.05 & 46.03 & 1.51 & 1.19 \\
\hline 500 & 9.57 & 48.35 & 150.33 & 111.10 & 9.36 & 48.71 & 20.71 & 16.77 \\
\hline 1000 & 9.79 & 48.94 & 1404.56 & 1163.49 & 9.80 & 48.92 & 137.39 & 119.29 \\
\hline
\end{tabular}

\begin{tabular}{|c|c|c|c|c|c|c|c|c|}
\hline \multirow[t]{3}{*}{$n$} & \multicolumn{8}{|c|}{ Multi-start } \\
\hline & \multicolumn{4}{|c|}{5 (Nofix-Full) } & \multicolumn{4}{|c|}{6 (Nofix-Half) } \\
\hline & $\mathrm{UB} \%$ & $\mathrm{LB} \%$ & $T$ & $T_{\text {best }}$ & $\mathrm{UB} \%$ & $\mathrm{LB} \%$ & $T$ & $T_{\text {best }}$ \\
\hline 10 & 2.44 & 33.04 & 0.00 & 0.00 & - & - & - & - \\
\hline 20 & 9.86 & 39.63 & 0.04 & 0.00 & 14.25 & 33.06 & 0.00 & 0.00 \\
\hline 50 & 9.74 & 45.30 & 3.54 & 3.14 & 9.67 & 45.39 & 1.91 & 1.77 \\
\hline 100 & 11.66 & 43.26 & 103.92 & 89.12 & 11.35 & 43.73 & 12.95 & 10.88 \\
\hline 200 & 16.21 & 34.96 & 3995.00 & 3752.26 & 16.24 & 34.92 & 476.41 & 409.33 \\
\hline 500 & 9.71 & 48.14 & 10381.36 & 9065.10 & 11.84 & 44.62 & 6918.79 & 6223.00 \\
\hline 1000 & - & - & - & - & - & - & - & - \\
\hline \multirow[t]{3}{*}{$n$} & \multicolumn{8}{|c|}{ Multi-start } \\
\hline & \multicolumn{4}{|c|}{$7($ Fix-Full) } & \multicolumn{4}{|c|}{ 8(Fix-Half) } \\
\hline & UB\% & LB\% & $T$ & $T_{\text {best }}$ & UB\% & $\mathrm{LB} \%$ & $T$ & $T_{\text {best }}$ \\
\hline$\overline{10}$ & 2.44 & 33.04 & 0.00 & 0.00 & - & - & - & - \\
\hline 20 & 9.41 & 40.34 & 0.01 & 0.00 & 14.15 & 33.08 & 0.00 & 0.00 \\
\hline 50 & 8.54 & 47.20 & 0.58 & 0.08 & 8.55 & 47.21 & 0.07 & 0.03 \\
\hline 100 & 11.00 & 44.28 & 3.97 & 2.95 & 9.79 & 46.15 & 0.94 & 0.69 \\
\hline
\end{tabular}


Table 6 continued

\begin{tabular}{|c|c|c|c|c|c|c|c|c|}
\hline \multirow[t]{3}{*}{$n$} & \multicolumn{8}{|c|}{ Multi-start } \\
\hline & \multicolumn{4}{|c|}{ 7(Fix-Full) } & \multicolumn{4}{|c|}{ 8(Fix-Half) } \\
\hline & UB\% & $\mathrm{LB} \%$ & $T$ & $T_{\text {best }}$ & $\mathrm{UB} \%$ & $\mathrm{LB} \%$ & $T$ & $T_{\text {best }}$ \\
\hline 200 & 13.86 & 38.77 & 82.37 & 68.03 & 13.02 & 40.08 & 18.70 & 13.97 \\
\hline 500 & 8.87 & 49.50 & 1825.40 & 1524.09 & 8.20 & 50.60 & 284.00 & 195.51 \\
\hline 1000 & 13.76 & 42.35 & 11031.62 & 9311.21 & 12.91 & 43.77 & 1906.01 & 1599.57 \\
\hline
\end{tabular}

Table 7 Results of the GRASP+VND Single-start on the TSP Library instances

\begin{tabular}{|c|c|c|c|c|c|c|c|}
\hline & \multirow[t]{2}{*}{$n$} & \multicolumn{3}{|c|}{1 (Nofix-Full) } & \multicolumn{3}{|c|}{2 (Nofix-Half) } \\
\hline & & $\mathrm{UB} \%$ & $\mathrm{LB} \%$ & $T$ & $\mathrm{UB} \%$ & $\mathrm{LB} \%$ & $T$ \\
\hline st70 & 70 & 4.94 & 27.66 & 2.14 & 6.62 & 26.50 & 0.23 \\
\hline att532 & 532 & 35.98 & 30.56 & 5134.36 & 31.98 & 32.6 & 555.27 \\
\hline kroD100 & 100 & 9.37 & 33.74 & 4.69 & 10.70 & 32.94 & 1.02 \\
\hline $\operatorname{lin} 105$ & 105 & 18.29 & 32.29 & 11.25 & 17.21 & 32.91 & 1.20 \\
\hline $\operatorname{lin} 318$ & 318 & 17.89 & 39.62 & 401.06 & 19.49 & 38.80 & 50.70 \\
\hline pr107 & 107 & 4.80 & 39.44 & 15.28 & 4.80 & 39.44 & 1.14 \\
\hline pr226 & 226 & 52.46 & 55.06 & 228.78 & 53.42 & 54.78 & 37.78 \\
\hline pr439 & 439 & 16.53 & 82.44 & 508.84 & 17.20 & 82.34 & 613.73 \\
\hline rat99 & 99 & 8.04 & 19.38 & 13.98 & 6.98 & 20.17 & 1.00 \\
\hline \multirow[t]{3}{*}{ rat195 } & 195 & 3.78 & 14.04 & 104.38 & 3.97 & 13.88 & 27.97 \\
\hline & $n$ & \multicolumn{3}{|c|}{3 (Fix-Full) } & \multicolumn{3}{|c|}{4 (Fix-Half) } \\
\hline & & $\mathrm{UB} \%$ & LB\% & $T$ & $\mathrm{UB} \%$ & LB\% & $T$ \\
\hline$\overline{\text { st70 }}$ & 70 & 3.24 & 28.83 & 0.08 & 8.18 & 28.83 & 0.02 \\
\hline att532 & 532 & 31.93 & 32.63 & 165.00 & 33.44 & 31.86 & 19.59 \\
\hline kroD100 & 100 & 9.01 & 33.97 & 0.41 & 8.83 & 34.07 & 0.08 \\
\hline $\operatorname{lin} 105$ & 105 & 13.84 & 34.84 & 0.34 & 12.17 & 35.79 & 0.11 \\
\hline $\operatorname{lin} 318$ & 318 & 16.25 & 40.46 & 8.30 & 16.14 & 40.52 & 2.80 \\
\hline pr107 & 107 & 4.80 & 39.44 & 0.16 & 4.80 & 39.44 & 0.03 \\
\hline pr226 & 226 & 53.07 & 54.88 & 5.34 & 53.94 & 54.62 & 0.53 \\
\hline pr439 & 439 & 15.26 & 82.63 & 125.56 & 15.65 & 82.57 & 23.33 \\
\hline rat99 & 99 & 4.39 & 22.10 & 0.24 & 3.42 & 22.82 & 0.03 \\
\hline rat195 & 195 & 3.51 & 14.26 & 7.20 & 3.51 & 14.26 & 0.28 \\
\hline
\end{tabular}

neighborhood descent or variable neighbourhood search improvement phase. Several neighborhood reduction schemes were also introduced to speed up the search. To test the approach, we generated a set of medium-sized and large instances and discussed an upper and a lower bound. Experiments show that our approach is able to solve the 
Table 8 Results of the GRASP+VND Multi-start on the TSPlib instances

\begin{tabular}{|c|c|c|c|c|c|c|c|}
\hline & \multirow[t]{2}{*}{$n$} & \multicolumn{3}{|c|}{5 (Nofix-Full) } & \multicolumn{3}{|c|}{6 (Nofix-Half) } \\
\hline & & UB\% & LB\% & $T$ & UB $\%$ & LB $\%$ & $T$ \\
\hline st70 & 70 & 5.26 & 27.44 & 22.24 & 6.62 & 26.50 & 2.23 \\
\hline att532 & 532 & 52.51 & 22.11 & 65121.06 & 43.29 & 26.82 & 5005.32 \\
\hline kroD100 & 100 & 11.29 & 32.59 & 54.69 & 11.16 & 32.66 & 11.02 \\
\hline $\operatorname{lin} 105$ & 105 & 19.04 & 31.85 & 101.27 & 17.41 & 32.79 & 12.23 \\
\hline $\operatorname{lin} 318$ & 318 & 22.92 & 37.04 & 3951.02 & 19.70 & 38.69 & 455.60 \\
\hline pr107 & 107 & 21.52 & 29.78 & 225.32 & 8.63 & 37.23 & 3.33 \\
\hline pr226 & 226 & 54.00 & 54.60 & 2328.71 & 53.42 & 54.78 & 239.56 \\
\hline pr439 & 439 & 23.13 & 81.45 & 4563.44 & 20.44 & 81.85 & 5614.74 \\
\hline rat99 & 99 & 9.77 & 18.09 & 195.31 & 6.98 & 20.17 & 9.00 \\
\hline \multirow[t]{3}{*}{ rat195 } & 195 & 4.56 & 13.39 & 884.78 & 4.46 & 13.48 & 311.97 \\
\hline & $n$ & \multicolumn{3}{|c|}{7 (Fix-Full) } & \multicolumn{3}{|c|}{8 (Fix-Half) } \\
\hline & & UB $\%$ & LB\% & $T$ & UB\% & LB\% & $T$ \\
\hline st70 & 70 & 7.45 & 25.93 & 1.10 & 6.28 & 30.07 & 1.11 \\
\hline att532 & 532 & 42.41 & 27.27 & 1065.09 & 27.89 & 34.69 & 317.09 \\
\hline kroD100 & 100 & 9.22 & 33.84 & 3.13 & 9.22 & 33.84 & 1.12 \\
\hline $\operatorname{lin} 105$ & 105 & 14.88 & 34.24 & 3.09 & 13.56 & 34.99 & 1.44 \\
\hline $\operatorname{lin} 318$ & 318 & 17.03 & 40.06 & 79.79 & 11.45 & 42.92 & 12.24 \\
\hline pr107 & 107 & 8.18 & 37.49 & 1.89 & 2.82 & 40.58 & 0.10 \\
\hline pr226 & 226 & 53.07 & 54.88 & 53.22 & 53.49 & 54.75 & 3.23 \\
\hline pr439 & 439 & 14.66 & 82.72 & 1256.31 & 15.20 & 82.64 & 196.22 \\
\hline rat99 & 99 & 6.01 & 20.89 & 2.95 & 6.01 & 20.89 & 1.02 \\
\hline rat195 & 195 & 1.99 & 15.52 & 55.46 & 2.93 & 14.74 & 1.05 \\
\hline
\end{tabular}

Table 9 Comparison of the best-found TRP-solution with the optimal TSP-solution using the TRP-objective function

\begin{tabular}{lrrrr}
\hline Problem & Optimal TSP & Best TRP & Difference & $\%$ Difference \\
\hline st70 & 22865 & 19553 & 3312 & 16.94 \\
att532 & 24186404 & 18448435 & 5737969 & 31.10 \\
kroD100 & 1072593 & 976830 & 95763 & 9.80 \\
lin105 & 904993 & 585823 & 319170 & 54.48 \\
lin318 & 7238644 & 5876537 & 1362107 & 23.18 \\
pr107 & 2040746 & 1983475 & 57271 & 2.89 \\
pr226 & 8284243 & 7226554 & 1057689 & 14.64 \\
pr439 & 19305544 & 18567170 & 738374 & 3.98 \\
rat99 & 60415 & 56994 & 3421 & 6.00 \\
rat195 & 226194 & 213371 & 12823 & 6.01 \\
Average & & & & 16.90 \\
\hline
\end{tabular}


generated instances, as well as a set of instances drawn from the TSP library, in a reasonable amount of time.

In the future, we intend to extend our algorithm by including more neighborhoods and carefully studying the effectiveness of each neighborhood on the TRP. We also intend to apply our algorithm to other customer-centric routing problems, involving more than one vehicle. Increasing the efficiency of our algorithm even more, to allow even larger problems to be solved, is another future research topic. Finally, the integration of exact methods for the TRP might lead to even better performing algorithms and is something that we plan to investigate.

While this paper was in the final stage of the review process, follow-up work was published by Ngeuveu et al. (2010). Although their focus is on the capacitated TRP, they suggest various procedures that would speed up the search for optimal solutions for the uncapacitated TRP and that, in general, lead to better solutions. Incorporation of such procedures in our GRASP+VNS/VND method is also left for future research.

Open Access This article is distributed under the terms of the Creative Commons Attribution Noncommercial License which permits any noncommercial use, distribution, and reproduction in any medium, provided the original author(s) and source are credited.

\section{References}

Afrati F, Cosmadakis S, Papadimitriou CH, Papageorgiou G, Papakostantinou N (1986) The complexity of the traveling repairman problem. Theor Inform Appl 20:79-87

Bianco L, Mingozzi A, Ricciardelli S (1993) The travelling salesman problem with cumulative costs. Networks 23(2):81-91

Blum A, Chalasani P, Coppersmith D, Pulleyblank B, Raghavan P, Sudan M (1994) The minimum latency problem. In: Proceedings of the twenty-sixth annual symposium on theory of computing (STOC), pp 163-171

Bräysy O, Gendreau M (2005) Vehicle routing problem with time windows, part II: metaheuristics. Transport Sci 39:119-139

Chaudhuri K, Godfrey B, Rao S, Talwar K (2003) Paths, trees, and minimum latency tours. In: Proceedings of 44th symposium on foundations of computer science (FOCS), pp 36-45

Christofides N (1976) Worst-case analysis of a new heuristic for the travelling salesman problem. Technical Report 388, Graduate School of Industrial Administration, Carnegie-Mellon University, Pittsburgh, PA

Cordone R, Wolfler Calvo R (2001) A heuristic for the vehicle routing problem with time windows. J Heuristics 7:107-129

Crispim J, Brandao J (2001) Reactive tabu search and variable neighborhood descent applied to the vehicle routing problem with backhauls. In: MIC 2001—Proceedings of the metaheuristics international conference, Porto, pp 631-636

Feo TA, Resende MGC (1989) A probabilistic heuristic for a computationally difficult set covering problem. Oper Res Lett 8:67-71

Feo TA, Resende MGC (1995) Greedy randomized adaptive search procedures. J Global Optim 6:109-133

Fischetti M, Laporte G, Martello S (1993) The delivery man problem and cumulative matroids. Oper Res 41(6):1055-1064

García A, Jodrá P, Tejel J (2002) A note on the traveling repairman problem. Networks 40(1):27-31

Hansen P, Mladenović N (1999) An introduction to variable neighborhood search. In: Voss S, Martello S, Osman I, Roucairol C (eds) Metaheuristics: advances and trends in local search paradigms for optimization. Kluwer, Boston pp 433-458

Hansen P, Mladenović N (2001a) Industrial applications of the variable neighbourhood search metaheuristic. In: Decisions and control in management science. Kluwer, Boston, pp 261-274 
Hansen P, Mladenović N (2001b) Variable neighbourhood search: principles and applications. Eur J Oper Res 130:449-467

Kontoravdis GA, Bard JF (1995) A GRASP for the vehicle routing problem with time windows. INFORMS J Comput 7:10-23

Mladenović N, Hansen P (1997) Variable neighbourhood search. Comput Oper Res 24:1097-1100

Méndez-Díaz I, Zabala P, Lucena A (2008) A new formulation for the traveling deliveryman problem. Discrete Appl Math 156(17):3223-3237

Ngeuveu SU, Prins C, Wolfler Calvo R (2010) An effective memetic algorithm for the cumulative capacitated vehicle routing problem. Comput OR 37(11):1877-1885

Ribeiro CC, Vianna DS (2003) A GRASP/VND heuristic for the phylogeny problem using a new neighborhood structure. Technical report, Department of Computer Science, Catholic U. of Rio de Janeiro, Rio de Janeiro, Brazil

Sarubbi J, Luna H (2007) A new flow formulation for the minimum latency problem. In: International network optimization conference (INOC), Spa, Belgium

Simchi-Levi D, Berman O (1991) Minimizing the total flow time of $n$ jobs on a network. IIE Trans 23(3):236-244

Wu BY (2000) Polynomial time algorithms for some minimum latency problems. Inform Process Lett 75:225-229

Wu BY, Huang Z-N, Zhan F-J (2004) Exact algorithms for the minimum latency problem. Inform Process Lett 92:303-309 